Results: Nine VBD patients ( 8 male and 1 female) were enrolled, with a mean age and median course of $37 \pm 8.6$ years and 72 months (range 12 to 300 ), respectively. Cardiac involvements (severe aortic regurgitation secondary to $\mathrm{BD})$ were presented in 7 patients, including 2 patients with post-operative paravalvular leakage (PVL) after aortic valve replacement surgery. Multiple vascular lesions were documented in the other 2 patients, including one patient with life-threatening multiple pulmonary aneurysms, pulmonary thromboembolism and recurrent deep vein thrombosis, and another patient with abdominal aortic pseudoaneurysm and multiple artery stenosis and occlusion. Prior to GOL therapy, all patients experienced disease progression despite high-dose glucocorticoids combined with multiple immunosuppressants. Moreover, seven patients required effective and fast control of inflammation and a decrease of glucocorticoid dose during the perioperative period. They were treated with GOL, 50mg every 4 weeks, in combination with background low-or medium-dose glucocorticoids and immunosuppressants, for a median of 6 (range 3-15) months. After a mean duration of follow-up of 10 (range 2-6) months, all patients achieved improvement both in clinical symptoms and serum inflammation markers. The ESR level $[4.88 \pm 4.94 \mathrm{~mm} / \mathrm{h}$ vs $31.13 \pm 31.78 \mathrm{~mm} / \mathrm{h}, \mathrm{P}<0.01]$ and CRP level $[1.9$ (0.11-3.73) $\mathrm{mg} / \mathrm{L}$ vs $24.3(0.4-85.57) \mathrm{mg} / \mathrm{L}, \quad \mathrm{P}<0.01]$ significantly decreased. The dosage of glucocorticoid[10 (0-15) vs $40(0-100) \mathrm{mg} / \mathrm{d}, \mathrm{P}<0.01]$ effectively tapered, indicating a potential steroid-sparing effect. No newly-onset aneurysm and recurrent venous thrombosis were observed. Also, one patient had a marked reduction in size and number of pulmonary aneurysms. No post-operative PVL was observed in the five patients after Bentall operation with a median follow-up of 10 months. One patient with severe aortic regurgitation remained stable and without surgical intervention with the treatment of GOL for 16 months. No severe complication occurred in one patient after underwent endovascular repair of abdominal aorta for 8 months. GOL was well-tolerated, and no serious adverse event was observed.

Conclusion: Our results suggested that GOL is safe and effective for the treatment of patients with severe and / or refractory VBD. Further controlled studies are warranted to confirm the therapeutic potential of GOL in VBD patients.

Disclosure of Interests: None declared

DOI: 10.1136/annrheumdis-2020-eular.2272

\section{AB0532 CORRELATION WITH THE FREQUENCY OF DISEASE RELAPSES DURING THE FIRST 3 YEARS FROM THE DIAGNOSIS AND DISEASE OUTCOMES IN BEHCET'S SYNDROME}

R. Talarico ${ }^{1}$, A. Figliomeni ${ }^{1}$, L. Mione ${ }^{1}$, A. Parma ${ }^{1}$, E. Cioffi ${ }^{1}$, M. Mosca ${ }^{1}$ on behalf of N/A. ${ }^{1}$ Rheumatology Unit, Department of Clinical and Experimental Medicine, University of Pisa, Pisa, Italy

Background: Beside the organ involvement, a number of demographic factors could considerably influence the long-term and short-term outcomes of Behçet's syndrome (BS): age at disease onset, duration of disease, gender and sex. Younger men patients are more suitable to have a more severe disease, due to an increased frequency both of morbidity and mortality, related to ocular,vascular and neurological involvement

Objectives: The primary aims of the study were to evaluate disease activity in a cohort of BS patients consecutively followed in a BS clinic of a tertiary centre and to explore whether there is a correlation between frequency of relapses in the first 3 years of diseases and disease outcomes.

Methods: One-hundred and sixty-five patients (91 males and 74 females; mean age $39 \pm 9$ years, mean disease duration $9 \pm 5$ ) with a diagnosis of BS according to the ISG criteria were studied. Disease activity has been evaluated by BDCAF and patients were also categorized in major or minor involvement of BS according or not to the presence of ocular, neurological and vascular involvement in the course of disease The numbers of relapses in the first 3 years from diagnosis were correlated with disease outcome and damage.

Results: At time of the evaluation, $47 \%$ of BS patients presented an active disease; 69 patients presented muco-cutaneous involvement, 39 ocular disease, 21 joint involvement, 12 neurological impairment and 9 gastro-enteric involvement. Seventy-nine percent of patients presented in the course of the disease a severe BS involvement and the majority was represented by patients characterised by a more frequent relapse in the first 3 years of disease (M/F: $65 / 48$, mean age $43 \pm 3$ years). Those patients who experienced a more higher number of relapse in the first 3 years compared to the others were also characterised by poor disease outcomes and worse prognosis over time and this correlation was independent by the therapies taken.

Conclusion: The high frequency of relapses during the first three years from diagnosis may be considered an important prognostic factor for disease outcome in BS patients, therefore could be taken into account as a useful element to tailor the management, not only according to the type and severity of symptoms and epidemiological factors.

Acknowledgments: none

Disclosure of Interests: None declared

DOI: 10.1136/annrheumdis-2020-eular.5004

\section{$\mathrm{AB} 0533$ \\ ANTI-NEUTROPHIL CYTOPLASMIC ANTIBODY (ANCA) IN GENERAL POPULATION WITHOUT ANCA} ASSOCIATED VASCULITIS

H. Tamaki ${ }^{1}$, S. Fukui ${ }^{1}$, T. Nakai ${ }^{1}$, G. Kidoguchi ${ }^{1}$, S. Kawaai ${ }^{1}$, H. Ozawa ${ }^{1}$, Y. Ikeda' ${ }^{1}$, A. Koido ${ }^{1}$, Y. Ohara ${ }^{1}$, H. Shimizu', K. Yamaguchi ${ }^{1}$, M. Okada ${ }^{1} .{ }^{1}$ St. Luke's International Hospital, Immuno-Rheumatology Center, Tokyo, Japan

Background: Currently it is hypothesized that many systemic autoimmune diseases occur due to environmental risk factors in addition to genetic risk factors. Anti-Neutrophil Cytoplasmic Antibody (ANCA) is mainly associated with three systemic autoimmune disease including granulomatosis with polyangiitis (GPA), microscopic polyangiitis (MPA), eosinophilic granulomatosis with polyangiitis (EGPA). It is known that ANCA can be positive before clinical symptoms in patients with known diagnosis of GPA and ANCA titers rise before clinical manifestations appear. However, prevalence of ANCA among general population is not well known. It has not been described as well how many of people with positive ANCA eventually develop clinical manifestations of ANCA associated Vasculitis.

Objectives: This study aims to estimate prevalence of ANCA in general population without ANCA associated Vasculitis. It also describes natural disease course of people with positive ANCA without ANCA associated Vasculitis. Risk factors for positive ANCA are also analyzed.

Methods: This is a single center retrospective study at Center for Preventive Medicine of St. Luke's International Hospital in Tokyo. ANCA was checked among the patients who wished to between 2018 and 2019. St. Luke's Health Check-up Database (SLHCD) was utilized to collect the data. The patients whose serum was measured for ANCA were identified. The data for basic demographics, social habits, dietary habits and laboratory data were extracted. The charts of the patients with positive ANCA were reviewed.

Results: Sera of total 1204 people were checked for ANCA. Of these 1204 people, $587(48.8 \%)$ are male and the mean age was 55.8 years (32.6 to 79). There were total 11 patients with positive ANCA. Myeloperoxidase ANCA (MPO-ANCA) was positive for 3 patients and proteinase 3 ANCA (PR3-ANCA) was positive for 8 patients. Of these 11 patients, 5 were male $(45.5 \%)$ and the mean age was 54.6 years. Two patients had history of autoimmune disease (primary biliary cirrhosis and ulcerative colitis). Five patients were evaluated by rheumatologists with the median follow-up period of 274 days. None of them developed clinical signs and symptoms of ANCA associated Vasculitis. Four out of five patients had ANCA checked later, two of which turned negative. The prevalence of ANCA in this cohort was $0.9 \%(95 \%$ confidence interva [95\% Cl]: $0.5 \%$ to $1.6 \%$ ). Univariate analysis was performed to identify risk factors of positive ANCA. The variables analyzed include age, gender, body mass index (BMI), smoking habits, alcohol intake, dietary habits (fruits, fish, red meat), hypertension, dyslipidemia, and laboratory data. None of these variables demonstrated statistically significant differences except for positive rheumatoid factor (ANCA positive group: $33 \%$ vs ANCA negative group: $9.1 \%$ $\mathrm{p}$ value $=0.044$ )

Conclusion: The prevalence of ANCA in this cohort was $0.9 \%(95 \% \mathrm{Cl}: 0.5 \%$ to $1.6 \%$ ). None of them who had a follow-up developed ANCA associated Vasculitis during the follow-up period. Longer follow-up and more patients are necessary to determine natural course of people with positive ANCA.

Disclosure of Interests: None declared DOI: 10.1136/annrheumdis-2020-eular.5075

\section{$\mathrm{AB} 0534$ \\ EFFICACY OF TOCILIZUMAB IN LARGE-VESSEL GIANT CELL ARTERITIS: A SINGLE-CENTER REAL- LIFE EXPERIENCE}

A. Tomelleri ${ }^{1}$, C. Campochiaro ${ }^{1}$, S. Sartorelli ${ }^{1}$, E. Baldissera ${ }^{1}$, L. Dagna ${ }^{1}$

${ }^{1}$ San Raffaele Hospital, Unit of Immunology, Rheumatology, Allergy and Rare diseases, Milan, Italy

Background: In a significant proportion of giant cell arteritis (GCA) patients, large vessels (LV) are affected ${ }^{1}$. GiACTA trial showed tocilizumab (TCZ) to be effective for the treatment of $\mathrm{GCA}^{2}$ but did not differentiate between patients with and without LV involvement and did not evaluate LV-imaging response.

Objectives: To assess efficacy of TCZ in LV-GCA, evaluating both clinical symptoms and vascular inflammation on PET scan. 
Methods: Data from GCA patients followed-up at our Institution between January 2003 and December 2019 were retrospectively collected. Only TCZtreated patients, with evidence of LV 18F-FDG-uptake at baseline (T0) and who repeated a PET scan after at least 6 months of treatment (T1) were included. All patients received subcutaneous TCZ $162 \mathrm{mg}$ weekly. PET scans were read by 2 physicians blinded with regard to clinical data and to each other's assessment; vascular inflammation was quantified using the PETVAS score ${ }^{3}$. Clinical symptoms, prednisone (PDN) dosage, and PETVAS score between T0 and T1 were compared. Flares and adverse events were recorded. Non parametric tests were used.

Results: 61 TCZ-treated GCA patients were identified. Of these, 28 (45.9\%) had evidence of vascular inflammation at T0, and 14 of them (10 female, mean age $66.2 \pm 7.4$ years) repeated a PET scan during follow-up, after a median of 13 months (IQR, 9.5-17.5). 10 patients started TCZ upon GCA relapse, with a median delay from GCA diagnosis of 7.5 (3-15.8) months; 4 patients were newly diagnosed and started TCZ at diagnosis. At T0, 10 patients had systemic symptoms, 6 patients had cranial symptoms, and 7 had polymyalgia rheumatica (PMR). 12/14 patients were on concomitant steroids, with a median PDN daily dose of 15 (15-25) mg. Erythrocyte sedimentation rate and C-reactive protein levels were respectively 35 (21-39) $\mathrm{mm} / \mathrm{h}$ and 7.8 (3.2-11) mg/L. Median PETVAS was 7 (3.3-11). At T1, 13/14 patients were asymptomatic; 1 patient had a PMR relapse. No GCA flare was recorded. Median PDN daily dose was $1.25(0-2.5), p=0.002$, and PDN suspension was achieved in 5 patients. Median PETVAS was $0(0-0.8), p=0.003$; only in 1 patient PETVAS increased between T0-T1 (from 2 to 5 ), in absence of clinical symptoms. This was the patient with the longest delay between diagnosis and TCZ start (124 months) and suspended TCZ for 3 months due to oral aphthosis. No patients had severe infections; 1 patient definitively suspended TCZ at T1 due to severe leukopenia.

Conclusion: In LV-GCA patients, TCZ seems to be effective in obtaining clinical remission and in reducing vascular inflammation on PET scan.

References:

[1] Dejaco C, et al. Nature Reviews Rheumatology (2017)

[2] Stone JH, et al. N Engl J Med (2017)

[3] Grayson PC, et al. Arthritis Rheumatol (2018)

Disclosure of Interests: Alessandro Tomelleri: None declared, Corrado Campochiaro Speakers bureau: Novartis, Pfizer, Roche, GSK, SOBI, Silvia Sartorelli: None declared, Elena Baldissera Speakers bureau: Novartis, Pfizer, Roche, Alpha Sigma, Sanofi, Lorenzo Dagna Grant/research support from: Abbvie, BMS, Celgene, Janssen, MSD, Mundipharma Pharmaceuticals, Novartis, Pfizer, Roche, SG, SOBI, Consultant of: Abbvie, Amgen, Biogen, BMS, Celltrion, Novartis, Pfizer, Roche, SG, and SOBI

DOI: 10.1136/annrheumdis-2020-eular.2022

\begin{tabular}{|l|l}
\hline AB0535 & ARE THE NEUTROPHIL/LYMPHOCYTE RATIO (NLR) \\
AND PLATELET/LYMPHOCYTE RATIO (PLR) USEFUL \\
TO COMPARED WITH ACUTE PHASE REACTANTS \\
(ESR/CRP) FOR DIAGNOSIS AND PROGNOSIS OF \\
PATIENTS WITH ACTIVE TAKAYASU'S ARTERITIS \\
(TAK)?: AN ANALYSIS BASED ON ROC AND \\
KAPLAN-MEIER CURVES IN A LATIN AMERICAN \\
POPULATION
\end{tabular}

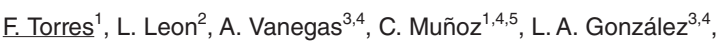
D. Jaramillo ${ }^{3,4,5} .{ }^{1}$ Universidad de Antioquia, Medellin, Colombia; ${ }^{2}$ Universidad Nacional de Colombia, Bogota, Colombia; ${ }^{3}$ Universidad de Antioquia, Medellin, Colombia; ${ }^{4}$ Hospital San Vicente Fundacion, Medellin, Colombia; ${ }^{5}$ Clinica Leon XIII IPS Universitaria, Medellin, Colombia

Background: The NIH criteria are the main measure to determine activity in TAK. NLR and PLR appear promising to predict disease activity ${ }^{(1)}$. TAK is one of the most frequent vasculitis in Colombia with a prevalence of $13.3 \%$; however, in more recent years the information is sparse about TAK clinical behavior in our region, which leads to a late diagnosis. Although survival has improved with immunosuppressive treatment, relapses remain high especially in the first year of diagnosis

Objectives: Compare NLR and PLR with ESR/CRP to predict TAK activity

Show survival and relapse in patients with TAK followed to $7 \mathrm{y}$

Methods: Retrospective cohort of 43 patients with TAK between 2011-2018 with prospective follow-up of relapses and mortality. 88\% fulfilled the ACR 1990 criteria. The disease activity was determined according to $\mathrm{NIH}$ criteria: active disease $(n=34)$ and inactive disease $(n=9)$. Through bivariate analysis, we compared the clinical and radiologic characteristics between age groups (table 1) using the Pearson test and Wilcoxon range test. Value of $p<0.05$ was statistically significant. Survival and relapse analysis were performed using Kaplan-Meier plots. Sensitivity $(\mathrm{Sn})$, specificity $(\mathrm{Sp})$ and area under the curve $(\mathrm{AUC})$ were
Table 1. Comparison of Clinical Features in Patients with TAK

\begin{tabular}{|c|c|c|c|}
\hline & \multicolumn{3}{|l|}{ No (\%) of Patients } \\
\hline & $\begin{array}{l}\text { Age at diagnosis } \\
\leq 40 \text { y }(n=32)\end{array}$ & $\begin{array}{c}\text { Age at } \\
\text { diagnosis } \\
\geq 40 \mathrm{y} \\
(\mathrm{n}=11)\end{array}$ & $\begin{array}{c}P \\
\text { value }\end{array}$ \\
\hline Female & $30(94)$ & $11(100)$ & 0.39 \\
\hline $\begin{array}{l}\text { Age at symptom onset (years), median: } \\
\text { (Interquartile range -IQR-) }\end{array}$ & $20(17-25)$ & $52(47-57)$ & 0.110 \\
\hline $\begin{array}{l}\text { Time from symptom onset to diagnosis (months) } \\
\text { median (IQR) }\end{array}$ & $9.5(2-15)$ & $36(1.5-24)$ & 0.774 \\
\hline Smoking & $2(6)$ & $6(54)$ & 0.002 \\
\hline Dyslipidemia & $7(22)$ & $6(54)$ & 0.042 \\
\hline \multicolumn{4}{|l|}{ ACR Criteria 1990} \\
\hline Claudication of extremities & $14(43)$ & $5(45)$ & 0.922 \\
\hline $\begin{array}{l}\text { Upper extremities blood pressure discrepancy > } \\
10 \mathrm{mmHg}\end{array}$ & $19(59)$ & $6(54)$ & 0.779 \\
\hline Decreased brachial artery pulse & $24(75)$ & $9(82)$ & 0.644 \\
\hline Subclavian artery or aorta bruit & $19(59)$ & $6(54)$ & 0.774 \\
\hline Arteriographic abnormality & $27 / 27(100)$ & $11 / 11(100)$ & 1.0 \\
\hline ESR (mm/h), median (IQR) & $56(23-115)$ & $38(33-76)$ & 0.011 \\
\hline CRP (mg/dL), median (IQR) & $1.35(0.4-3.4)$ & $3(1.49-18.5)$ & 0.020 \\
\hline Creatinine (mg/dL), median (IQR) & $0.72(0.6-0.83)$ & $0.84(0.75-1)$ & 0.007 \\
\hline \multicolumn{4}{|l|}{ Hata-Numano classification } \\
\hline I & $15 \%$ & $18 \%$ & \\
\hline Ila & $6 \%$ & $9 \%$ & \\
\hline Ilb & $6 \%$ & & \\
\hline III & $6 \%$ & & \\
\hline IV & $3 \%$ & $9 \%$ & \\
\hline v & $59 \%$ & $64 \%$ & \\
\hline
\end{tabular}

determined for NLR, PLR, ESR and CRP by receiver-operating curves (ROC) compared to NIH criteria.

Results: 41 patients were women (96\%) with a median age at diagnosis of 22 $y$ and an interval from the onset of disease to diagnosis of 12 months (IR:1$168 \mathrm{~m}$ ). The population over 40 years had a greater comorbidities burden (54\% had history of smoking and dyslipidemia) and a major interval between the onset of disease and the diagnosis (36 months vs 9.5 months). Most frequent vascular phenotypes were types V (62\%) and I (16\%). NLR and PLR showed poor performance to predict activity compared with CRP; NLR level of 1.74 showed to be the predictive cut-off value for active TAK (Sn: $85.3 \%$, Sp: $37.5 \%, A U C=0.563)$. PLR level of 112.5 was found to be the predictive cut-off value for active TAK (Sn: $76.5 \%, \mathrm{Sp}: 50 \%, \mathrm{AUC}=0.517$ ). The CRP was the most accurate biomarker ( $\mathrm{Sn}: 79.4 \%, \mathrm{Sp}: 75 \%, \mathrm{AUC}=0.761)$ while the $\mathrm{ESR}$ was lower to predict activity (Sn: $63.6 \%, \mathrm{Sp}: 75 \%, \mathrm{AUC}=0.598)$ (figure 1). At 5 years, survival was $83 \%$ and $50 \%$ of patients had presented at least one relapse (figure 2)

Conclusion: Our data does not support the use of NLR or PLR to differentiate relapse and remission in TAK. CRP had better diagnostic performance than ESR in the prediction of activity compared to NIH criteria. The 5-year survival in this cohort is below that reported after 1985 (reported survival: 90-96\%) ${ }^{(2)}$
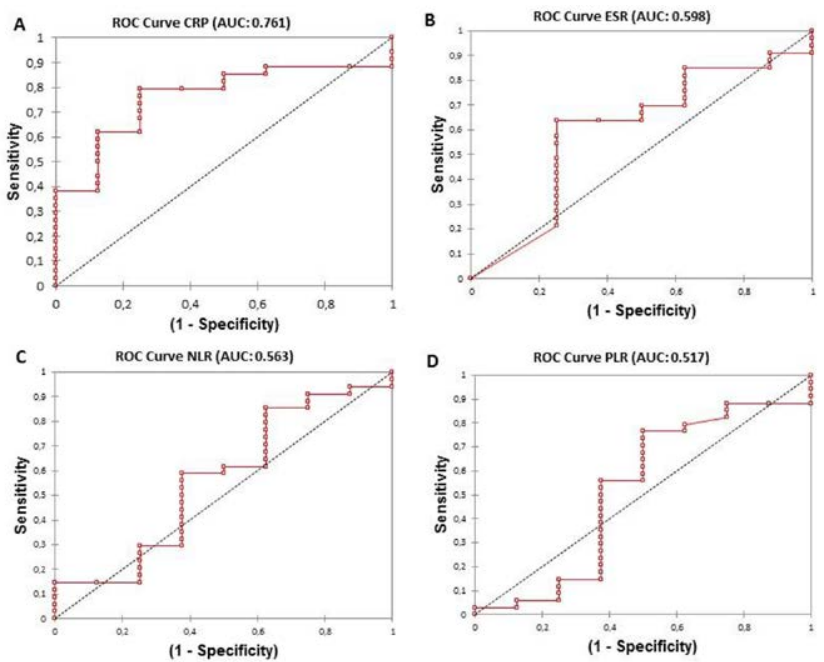

Figure 1: Receiver-operating characteristic (ROC) C
ratio (NLR) and D: Platelet-to-lymphocyte ratio (PLR) 